rates as other commercially available catheters with no safety concerns. More prospective clinical data is needed to fully assess the Q Aspiration Catheter.

Abbreviations AIS (acute ischemic stroke), MT (mechanical thrombectomy), ASPECTs (Alberta Stroke Program Early CT Score), mTICI (modified TICI score), NIHSS (National Institute of Health Stroke Scale), mRS (modified Rankin Scale), ENT (embolization to a new territory), sICH (symptomatic intracranial hemorrhage)

Disclosures F. Ballenilla: 2; C; MIVI Neuroscience, Inc. M. Espinosa: None. J. Zamarro: None. J. Masso: None. E. Garmendia: None. J. Pumar: None. J. Larrea: None. A. Mosqueira: None.

\section{P-013 NAVIGATING THE WALRUS: A LARGE BORE BALLOON GUIDE CATHETER FOR ACUTE ISCHEMIC STROKE THROMBECTOMY}

${ }^{1} \mathrm{GM}$ Cortez ${ }^{*},{ }^{2} \mathrm{RD}$ Turner, ${ }^{3} \mathrm{~A}$ Monteiro, ${ }^{4} \mathrm{AS}$ Puri, ${ }^{3} \mathrm{AH}$ Siddiqui, ${ }^{5} \mathrm{~J}$ Mocco, ${ }^{2} \mathrm{~J}$ Vargas, ${ }^{4} \mathrm{AL}$ Kuhn, ${ }^{5} \mathrm{~S}$ Majidi, ${ }^{2} \mathrm{Ml}$ Chaudry, ${ }^{1} \mathrm{~A}$ Aghaebrahim, ${ }^{2} \mathrm{AS}$ Turk, ${ }^{1} \mathrm{E}$ Sauvageau, ${ }^{1} \mathrm{R}$ Hanel. ${ }^{1}$ Neurosurgery, Baptist Health System- Jacksonville, Jacksonville, FL; ${ }^{2}$ Neurosurgery, Prisma Health Upstate, Greenville, SC; ${ }^{3}$ Neurosurgery, University at Buffalo, Buffalo, NY; ${ }^{4}$ Radiology, University of Massachusetts Medical Center, Worcester, MA; ${ }^{5}$ Neurosurgery, Icahn School of Medicine at Mount Sinai, New York, NY

\subsection{6/neurintsurg-2021-SNIS.49}

Introduction The utilization of Balloon-guide catheter (BCG) in acute ischemic stroke treatment has been associated with increased rates of successful recanalization, first-pass effect, and reduced procedural times. The Walrus BGC is the first in class, $0.087^{\prime}$ inner diameter and variable stiffness, designed to overcome some of the early technical constraints associated with previous models of BGCs. We aim to evaluate the performance and safety of the Walrus BGC.

Methods A retrospective review of 5 US comprehensive stroke centers was performed between August-2019 and December2020. Consecutive patients who underwent mechanical thrombectomy using the Walrus BGC were included. Performance analysis included the ability to track the device and enable therapeutic delivery. Baseline characteristics, procedural outcomes, adverse events, and functional outcomes were retrieved.

Results A total of 338 patients underwent mechanical thrombectomy using the Walrus BGC. The Walrus was successfully tracked into distal vasculature and allowed therapeutic device delivery in $99.1 \%$ of cases. Regarding thrombectomy first-pass modality, stent-retriever was used in 59.2\% (200/338); aspiration on 138 (40.8\%). Twenty-two different intermediate catheters were successfully accommodated into the Walrus BGC, with large ID $\geq 0.070$ ' aspiration catheters used in $71.9 \%$ $(243 / 338)$ of the cases. Successful vessel recanalization (mTICI$2 \mathrm{~b} / 3$ ) was achieved in $94.4 \%$ (319/338), with a rate of firstpass mTICI-2b/3 of $64.8 \%$ (219/338). Mean puncture-torecanalization was $37.5 \pm 25 \mathrm{~min}$, and a median number of passes was 1 (IQR,1-2). Walrus-related adverse event was $0.6 \%$, corresponding to 2 vessel dissections. Functional independence was $50 \%(161 / 252)$ and mortality $25 \%(63 / 252)$. Unfavorable outcomes were more likely in patients older than 80 yo, unsuccessful reperfusion, longer procedure times, and a higher number of passes $(\mathrm{p}<0.05)$.

Conclusion The Walrus BGC presents as a safe surrogate to previous BGCs, demonstrating good trackability, a large inner diameter that allows use of largest available aspiration catheters, with a great safety profile. Procedural outcomes are in equipoise with historical data.

Disclosures G. M. Cortez: None. R. D. Turner: 2; C; Q’Apel. A. Monteiro: None. A. S. Puri: 2; C; Q'Apel. A. H. Siddiqui: 2; C; Q’Apel. J. Mocco: 2; C; Q’Apel. J. Vargas: None. A. L. Kuhn: None. S. Majidi: None. M. I. Chaudry: 2; C; Q'Apel. A. Aghaebrahim: None. A. S. Turk: 2; C; Q'Apel. E. Sauvageau: None. R. Hanel: 2; C; Q'Apel.

\section{P-014 REPERFUSION CATHETER MALFUNCTION DURING STROKE INTERVENTION: AN ANALYTICAL REVIEW OF THE FDA'S MAUDE DATABASE}

D Bageac*, B Gershon, R De Leacy. The Mount Sinai Hospital, New York, NY

\subsection{6/neurintsurg-2021-SNIS.50}

Background In December 2020, the JET7 Xtra Flex reperfusion catheter was voluntarily recalled after reports of injury and death associated with device malfunction. The FDA's MAUDE (Manufacturer and User Facility Device Experience) database aggregates reports of adverse events and product problems involving medical devices. We present the first analytical review of all MAUDE reports involving FDA cleared reperfusion catheters in order to summarize safety events to date and characterize unique modes of device failure.

Methods All publicly available MAUDE datasets were downloaded and linked, and all events associated with catheters carrying product code 'NRY' were extracted. Product code 'NRY' encompasses all devices intended to restore blood flow by removing thrombus/clots in patients experiencing ischemic stroke. Note that this review does not include catheters used off-label for aspiration thrombectomy.

Event characteristics including device type, malfunction and adverse events were collected directly as reported on MedWatch Form 3500. In order to characterize procedure-specific details, narrative information submitted in MedWatch Form 3500 Sections $\mathrm{B}$ and $\mathrm{H}$ was coded by 2 independent raters.

Results Between 1/1/2003 and 8/31/2020, there were 1,808 reperfusion catheter defects and/or malfunctions reported in the MAUDE database. Of these, $93.4 \%$ did not result in any adverse effect on patients. There were 112 malfunctions associated with an adverse event, of which 98 (87.5\%) resulted in injury, and $14(12.5 \%)$ resulted in death. A summary of malfunctions is available in table 1 . When interpreting these findings, it is important to note that FDA surveillance of these devices does not include data on the number of units sold, thus event rates cannot be determined or compared across devices.

Of 14 reported deaths, 13 involved malfunctions of the Jet 7 Xtra Flex catheter. All of these cases reported mechanical failure at the tip of the catheter, 10 of which involved tip rupture or ballooning following contrast injection. Ballooning was unique to the Jet $7 \mathrm{Xtra}$ Flex, and was also responsible for 3 patient injuries and 37 cases of malfunction that did not result in adverse outcomes.

One death and 45 injuries involved the 3 MAX Reperfusion catheter. All of these events were caused by catheter fracture leading to fragment embolization or vessel injury. This mechanism of failure was not unique to the $3 \mathrm{MAX}$, and was present in $61.2 \%$ of all malfunctions resulting in injury.

Among malfunctions that did not result in adverse events, the majority (59.1\%) were attributable to damage that 